\title{
Regulation and Roles of Autophagy in the Brain
}

\author{
Nektarios Tavernarakis
}

Autophagy is crucial for neuronal integrity. Loss of key autophagic components leads to progressive neurodegeneration and structural defects in neuronal synapses. However, the molecular mechanisms regulating autophagy in the brain remain elusive. Similarly, while it is widely accepted that protein turnover is required for synaptic plasticity, the contribution of autophagy to the degradation of synaptic proteins is unknown. We find that BDNF signaling via the tropomyosin receptor kinase $\mathrm{B}$ (TrkB) and the phosphatidylinositol 3-kinase (PI3K)/Akt pathway suppresses autophagy in vivo. Autophagy is differentially regulated by fasting, in different brain regions. Suppression of autophagy is required for BDNF-induced synaptic plasticity and for memory enhancement, under conditions of nutritional stress. BDNF signaling suppresses autophagy in the forebrain of adult mice. Indeed, BDNF ablation in the neural lineage causes uncontrolled increase in autophagy. In turn, increased autophagy mediates the synaptic defects caused by BDNF deficiency. Thus, fasting suppresses autophagy in regions of the mouse forebrain, thereby promoting synaptic remodeling and memory through a BDNF-regulated mechanism. We identify three key remodelers of postsynaptic densities as cargo of autophagy. Our results establish autophagy as a pivotal component of BDNF signaling, which is essential for BDNFinduced synaptic plasticity. This molecular mechanism underlies behavioral adaptations that increase fitness in times of scarcity.

\footnotetext{
N. Tavernarakis $(\bowtie)$

Institute of Molecular Biology and Biotechnology, Foundation for Research and TechnologyHellas \& Medical School, University of Crete, Heraklion, Greece

e-mail: tavernarakis@imbb.forth.gr
} 